\title{
A New Extremely Flexible Version of The Exponentiated Weibull Model: Theorem and Applications to Reliability and Medical Data Sets
}

\author{
Mohamed Aboraya \\ Department of Applied Statistics and Insurance, \\ Damietta University, Egypt. \\ Mohamedaboraya17@gmail.com
}

\begin{abstract}
In this work, a new lifetime model is introduced and studied. The major justification for the practicality of the new model is based on the wider use of the exponentiated Weibull and Weibull models. We are also motivated to introduce the new lifetime model since it exhibits decreasing, upside down-increasing, constant, increasing-constant and $\mathbf{J}$ shaped hazard rates also the density of the new distribution exhibits various important shapes. The new model can be viewed as a mixture of the exponentiated Weibull distribution. It can also be considered as a suitable model for fitting the symmetric, left skewed, right skewed and unimodal data. The importance and flexibility of the new model is illustrated by four read data applications.
\end{abstract}

Keywords: Burr-Hatke Differential Equation; Exponentiated Weibull; Maximum Likelihood Estimation; Generating Function; Moments.

\section{Genesis of the new model}

A random variable (R.V.) $T$ is said to have the Exponentiated Weibull (EW) distribution (see Mudholkar and Srivastava (1993), Mudholkar et al., (2015) and Nadarajah et al., (2013)) if its probability density function (P.D.F.), cumulative distribution function (C.D.F.) and the reliability function (R.F.) are given by

$$
\begin{gathered}
g_{E W}(t ; \alpha, \beta)=\alpha \beta t^{\beta-1}\left[1-\exp \left(-t^{\beta}\right)\right]^{\alpha-1} \exp \left(-t^{\beta}\right), \\
G_{E W}(t ; \alpha, \beta)=\left[1-\exp \left(-t^{\beta}\right)\right]^{\alpha},
\end{gathered}
$$

and

$$
R_{E W}(t)=\bar{G}_{E W}(t ; \alpha, \beta)=1-G_{E W}(t ; \alpha, \beta)=\left\{1-\left[1-\exp \left(-t^{\beta}\right)\right]^{\alpha}\right\},
$$

respectively, for $t>0, \alpha>0$ and $\beta>0$. When $\alpha=1$ we get the standard one parameter W model (see Weibull (1951)). In statistical literature, the Burr-Hatke differential equation (B.H.D.E.) can be written as

$$
d F / d t=g(t, F) F(1-F) \text { with } F_{0}=\left.F\left(t_{0}\right)\right|_{\left[t_{0} \in \Re\right]},
$$

where $F(t)=F$ is the C.D.F. of a continuous R.V. $T$ and $g(t, F)$ is an arbitrary positive function $\left(g^{(+)}\right)$for any $t_{0} \in \Re$. Using (1), Maniu and Voda (2008) introduced and studied the BH distribution with C.D.F. and P.D.F. given by

$$
F(t ; \theta)=1-\left.\frac{(t+1)^{-1}}{\exp (t \theta)}\right|_{[t>0, \theta>0]}
$$

and

$$
f(t ; \theta)=\frac{(t+1)^{-2}[\theta(t+1)+1]}{\exp (t \theta)}
$$


respectively. By replacing $t$ by $\left\{-\log \left[\bar{G}_{E W}(x)\right]\right\}$, Yousof et al., (2018) introduced a new flexible family of distributions called the BH-G family of distributions. Based on Yousof et al., (2018), the C.D.F. of the BHEW distribution can be defined as

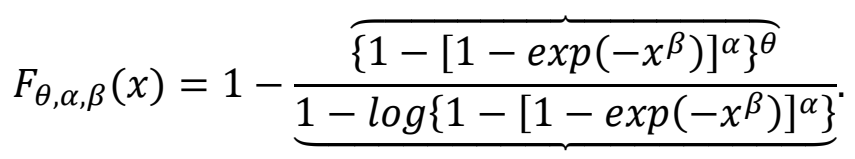

Equation (2) can be also obtained using idea of Alzaatreh et al., (2013). The P.D.F. corresponding to (2) is given by

$$
\begin{aligned}
f_{\theta, \alpha, \beta}(x)= & f(x ; \theta, \alpha, \beta)=\alpha \beta x^{\beta-1} \exp \left(-x^{\beta}\right)\left[1-\exp \left(-x^{\beta}\right)\right]^{\alpha-1} \\
& \times \frac{\left\{1-\left[1-\exp \left(-x^{\beta}\right)\right]^{\alpha}\right\}^{\theta-1}}{\left(1-\log \left\{1-\left[1-\exp \left(-x^{\beta}\right)\right]^{\alpha}\right\}\right)^{2}} \\
\times & {\left[\theta\left(1-\log \left\{1-\left[1-\exp \left(-x^{\beta}\right)\right]^{\alpha}\right\}\right)+1\right] . }
\end{aligned}
$$

The R.F. and hazard rate function (H.R.F.) of new model are given by

$$
R_{\theta, \alpha, \beta}(x)=\frac{\left\{1-\left[1-\exp \left(-x^{\beta}\right)\right]^{\alpha}\right\}^{\theta}}{1-\log \left\{1-\left[1-\exp \left(-x^{\beta}\right)\right]^{\alpha}\right\}^{\prime}}
$$

and

$$
\begin{aligned}
& h_{\theta, \alpha, \beta}(x)=\frac{\alpha \beta x^{\beta-1} \exp \left(-x^{\beta}\right)\left[1-\exp \left(-x^{\beta}\right)\right]^{\alpha-1}}{\left\{1-\left[1-\exp \left(-x^{\beta}\right)\right]^{\alpha}\right\}\left[1-\log \left\{1-\left[1-\exp \left(-x^{\beta}\right)\right]^{\alpha}\right\}\right]} \\
& \times\left[\theta\left(1-\log \left\{1-\left[1-\exp \left(-x^{\beta}\right)\right]^{\alpha}\right\}\right)+1\right] .
\end{aligned}
$$

Some useful extension of the $\mathrm{W}$ and EW models are developed by Yousof et al., (2015), Aryal et al. (2017), Yousof et al., (2017), Brito et al., (2017), Hamedani et al., (2017), Aboraya (2018), Almamy et al., (2018), Cordeiro et al., (2018), Korkmaz et al., (2019), among others.

\section{Justification}

The major justification for the practicality of the new model is based on the wider use of the exponentiated Weibull and Weibull models. We are also motivated to introduce the new lifetime model since it exhibits decreasing, unimodal and constant hazard rates (see Figure 2) also the P.D.F. of the new distribution exhibits various important shapes such as decreasing, unimodal, right skewed and left skewed (see Figure 1). The new model can be viewed as a mixture of the EW distribution. It can also be considered as a suitable model for fitting the symmetric, left skewed, right skewed, and unimodal data (see application section).

The proposed lifetime model is better than the Poisson Topp Leone-Weibull, Marshall Olkin extended-Weibull, Gamma-Weibull, Kumaraswamy-Weibull, Weibull-Fréchet, Beta-Weibull, Kumaraswamy transmuted-Weibull, transmuted modified-Weibull, transmuted exponentiated generalized Weibull, modified beta-Weibull, McDonaldWeibull models in modeling the failure times data. In modeling cancer patient's data, the new model is much better than the transmuted linear exponential, Weibull, Transmuted 
modified-Weibull, modified beta-Weibull, transmuted additive-Weibull, exponentiated transmuted generalized Rayleigh models. The new model is much better than the Weibull-Weibull, Odd Weibull-Weibull, gamma exponentiated-exponential models in modeling survival times of Guinea pigs. Finally, the proposed model is much better than the exponentiated-Weibull, transmuted-Weibull, Odd Log Logistic-Weibull models in modeling glass fibers data. Figure 1 shows that the P.D.F. BHEW distribution exhibits various important shapes such as decreasing, unimodal, right skewed and left skewed, from Figure 2 we conclude that the H.R.F. of the BHEW distribution exhibits decreasing, unimodal and constant hazard rates (Figure 1 and 2 are given in Appendix).

\section{Properties}

\subsection{Asymptotic}

Proposition Let $a=\inf \left\{x \mid G_{E W}(x)>0\right\}$. The asymptotics of the C.D.F., P.D.F. and H.R.F. as $x \rightarrow$ a are:

and

$$
\begin{aligned}
& \left.F_{\theta, \alpha, \beta}(x) \sim\left[1-\exp \left(-x^{\beta}\right)\right]^{\alpha}\right|_{[x \rightarrow a]}, \\
& \left.f_{\theta, \alpha, \beta}(x) \sim \alpha \beta x^{\beta-1} \exp \left(-x^{\beta}\right)\left[1-\exp \left(-x^{\beta}\right)\right]^{\alpha-1}\right|_{[x \rightarrow a]},
\end{aligned}
$$

$$
\left.h_{\theta, \alpha, \beta}(x) \sim \alpha \beta x^{\beta-1} \exp \left(-x^{\beta}\right)\left[1-\exp \left(-x^{\beta}\right)\right]^{-1}\right|_{[x \rightarrow a]}
$$

Proposition The asymptotic of C.D.F., P.D.F. and H.R.F. as $\mathrm{x} \rightarrow \infty$ are:

and

$$
\begin{aligned}
& 1-\left.F_{\theta, \alpha, \beta}(x) \sim \theta\left\{1-\left[1-\exp \left(-x^{\beta}\right)\right]^{\alpha}\right\}^{\theta}\right|_{[x \rightarrow \infty]}, \\
& f_{\theta, \alpha, \beta}(x) \sim \theta^{2} \alpha \beta x^{\beta-1} \exp \left(-x^{\beta}\right)\left\{1-\left[1-\exp \left(-x^{\beta}\right)\right]^{\alpha}\right\}^{\theta-1}[1 \\
& \left.-\exp \left(-x^{\beta}\right)\right]\left.^{\alpha-1}\right|_{[x \rightarrow \infty]},
\end{aligned}
$$

$$
\left.h_{\theta, \alpha, \beta}(x) \sim \theta \alpha \beta x^{\beta-1} \exp \left(-x^{\beta}\right)\left\{1-\left[1-\exp \left(-x^{\beta}\right)\right]^{\alpha}\right\}^{-1}\left[1-\exp \left(-x^{\beta}\right)\right]^{\alpha-1}\right|_{[x \rightarrow \infty]} \text {. }
$$

\subsection{Useful expansions}

Consider the following expansions

And

$$
(-z+1)^{t}=\left.\sum_{d=0}^{\infty}(-1)^{d}\left(\begin{array}{l}
t \\
d
\end{array}\right) z^{t}\right|_{[|z|<1]},
$$

$$
\log (-z+1)=-\left.\sum_{w=0}^{\infty}\left[z^{w+1} /(w+1)\right]\right|_{[|z|<1]} .
$$

Applying (4) for $A$ in Eq. (2) we get

$$
\left\{-\left[1-\exp \left(-x^{\beta}\right)\right]^{\alpha}+1\right\}^{\theta}=\sum_{d=0}^{\infty} a_{d}\left\{\left[1-\exp \left(-x^{\beta}\right)\right]^{\alpha}\right\}^{d},
$$

where 


$$
a_{d}=(-1)^{d}\left(\begin{array}{l}
\theta \\
d
\end{array}\right)
$$

Applying (5) for the term $B$, still in Eq. (2), we obtain

$$
\begin{aligned}
& 1-\log \left\{-\left[1-\exp \left(-x^{\beta}\right)\right]^{\alpha}+1\right\}=1+\sum_{i=0}^{\infty} \frac{\left\{\left[1-\exp \left(-x^{\beta}\right)\right]^{\alpha}\right\}^{i+1}}{(i+1)} \\
& =\sum_{d=0}^{\infty} b_{d}\left\{\left[1-\exp \left(-x^{\beta}\right)\right]^{\alpha}\right\}^{d},
\end{aligned}
$$

where $b_{0}=1$ and for $d \geq 1, b_{d}=\frac{-1}{d=0}$. Then, Eq. (2) can be writen as

$$
\begin{aligned}
& F_{\theta, \alpha, \beta}(x)=1-\frac{\sum_{d=0}^{\infty} a_{d}}{\sum_{\infty}^{\infty} b_{d}} \frac{\left\{\left[-\exp \left(-x^{\beta}\right)\right]^{\alpha}+1\right\}^{d}}{\left\{\left[-\exp \left(-x^{\beta}\right)\right]^{\alpha}+1\right\}^{d}} \\
& =1-\sum_{d=0}^{\infty} c_{d}\left\{\left[1-\exp \left(-x^{\beta}\right)\right]^{\alpha}\right\}^{d}
\end{aligned}
$$

where

$$
c_{0}=\frac{a_{o}}{b_{0}}
$$

and, for $d \geq 1$, we have

$$
c_{d}=\left(a_{d}-\frac{1}{b_{0}} \sum_{r=1}^{d} b_{r} c_{d-r}\right) / b_{0} .
$$

At the end, the C.D.F. (2) can be written as

$$
F_{\theta, \alpha, \beta}(x)=\sum_{d=0}^{\infty} V_{d+1} \Pi_{(d+1) \alpha}(x),
$$

where $d_{0}=1-c_{d}$, for $d \geq 1$ we have $d_{0}=-c_{d}$ and

$$
\left[-\exp \left(-x^{\beta}\right)+1\right]^{(d+1) \alpha}=\Pi_{(d+1) \alpha}(x)
$$

is the C.D.F. of the Exp-G family with power parameter $(d+1) \alpha$. By differentiating (6), we obtain the same mixture representation

$$
f_{\theta, \alpha, \beta}(x)=\sum_{d=0}^{\infty} V_{d+1} \pi_{(d+1) \alpha}(x ; \xi),
$$

where

$$
\begin{aligned}
\pi_{(d+1) \alpha}(x)= & {[(d+1) \alpha] \alpha \beta x^{\beta-1} \exp \left(-x^{\beta}\right)\left[1-\exp \left(-x^{\beta}\right)\right]^{(d+1) \alpha-1}[1} \\
& \left.-\exp \left(-x^{\beta}\right)\right]^{\alpha-1}
\end{aligned}
$$

is the EW P.D.F. with power parameter $(d+1) \alpha$. Eq. (7) means that the BHEW function is a linear combination of EW densities. So that, some the structural properties of the new model can be immediately obtained from the well-established properties of the EW distribution. 


\subsection{Moments and generating function}

The $r^{\text {th }}$ ordinary moment of $X$ is given by

Then, we obtain

$$
\mu_{r}^{\prime}=E X^{r}=\int_{-\infty}^{\infty} x^{r} f_{\theta, \alpha, \beta}(x) d x .
$$

$$
\mu_{r}^{\prime}=\left.\Gamma\left(1+r \beta^{-1}\right) \sum_{d, m=0}^{\infty} \xi_{d, m}^{((d+1) \alpha, r)}\right|_{[r>-\beta]},
$$

where

and

$$
V_{d+1} \xi_{m}^{((d+1) \alpha, r)}=\xi_{d, m}^{((d+1) \alpha, r)}
$$

$$
\xi_{\tau}^{(\eta, q)}=\eta(-1)^{\tau}(\tau+1)^{-(q+\beta) / \beta}\left(\begin{array}{c}
\eta-1 \\
\tau
\end{array}\right)
$$

Setting $r=1,2,3$ and 4 we get

$$
\begin{aligned}
& E X=\left.\Gamma(1+1 / \beta) \sum_{d, m=0}^{\infty} \xi_{d, m}^{(d+1) \alpha, 1)}\right|_{[1>-\beta]}, \\
& E X^{2}=\left.\Gamma(1+2 / \beta) \sum_{d, m=0}^{\infty} \xi_{d, m}^{((d+1) \alpha, 2)}\right|_{[2>-\beta]}, \\
& E X^{3}=\left.\Gamma(1+3 / \beta) \sum_{d, m=0}^{\infty} \xi_{d, m}^{((d+1) \alpha, 3)}\right|_{[3>-\beta]},
\end{aligned}
$$

and

$$
E X^{4}=\left.\Gamma(1+4 / \beta) \sum_{d, m=0}^{\infty} \xi_{d, m}^{((d+1) \alpha, 4)}\right|_{[4>-\beta]} .
$$

\subsection{Effect of $\boldsymbol{\theta}$ on the mean, variance, skewness and kurtosis}

From Table 1 we note that:

1- EX decreases as $\theta$ increases.

2- The variance decreases as $\theta$ increases.

3 - The new mode will be more skewed to the right as $\theta$ increases.

4-The kurtosis of new mode increases as $\theta$ increases. 
Table 1: Mean, variance, skewness and kurtosis.

\begin{tabular}{|c|c|c|c|c|c|c|}
\hline$\theta$ & $\alpha$ & $\beta$ & $\mathbf{E} X$ & Variance & Skewness & Kurtosis \\
\hline 5 & 0.5 & 0.5 & 0.00721373 & 0.001326813 & 19.46052 & 849.4605 \\
\hline 6 & & & 0.004238804 & 0.000488343 & 20.70598 & 979.9014 \\
\hline 7 & & & 0.00266581 & 0.0002043993 & 21.86453 & 1111.439 \\
\hline 8 & & & 0.001766359 & $9.430646 \times \mathrm{e}^{-05}$ & 22.94609 & 1243.363 \\
\hline 9 & & & 0.001219828 & $4.697908 \times \mathrm{e}^{-05}$ & 23.95796 & 1375.071 \\
\hline 10 & & & 0.0008711163 & $2.490317 \times \mathrm{e}^{-05}$ & 24.90484 & 1505.966 \\
\hline 15 & & & 0.0002271622 & $1.999047 \times \mathrm{e}^{-06}$ & 28.12918 & 2067.223 \\
\hline 20 & & & $6.501137 \times \mathrm{e}^{-05}$ & $3.060367 \times \mathrm{e}^{-07}$ & 30.54657 & 2569.744 \\
\hline 30 & & & $7.538604 \times \mathrm{e}^{-06}$ & $1.941787 \times \mathrm{e}^{-08}$ & 34.60131 & 3399.615 \\
\hline 50 & & & $1.00447 \times \mathrm{e}^{-07}$ & $2.167821 \times \mathrm{e}^{-10}$ & 152.5896 & 28718.54 \\
\hline 100 & & & $1.281096 \times \mathrm{e}^{-12}$ & $2.742489 \times \mathrm{e}^{-15}$ & 40878.74 & 1671225019 \\
\hline 150 & & & $1.233945 \times \mathrm{e}^{-17}$ & $2.641542 \times \mathrm{e}^{-20}$ & 13171437 & $1.734868 \times \mathrm{e}^{14}$ \\
\hline
\end{tabular}

The moment generating function M.G.F.can be derived via Eq. (7) as

where

$$
m_{r}(y)=\left.\Gamma\left(1+r \beta^{-1}\right) \sum_{d, r, m=0}^{\infty} \xi_{d, r, m}^{((d+1) \alpha, r)}\right|_{[r>-\beta]},
$$

$$
\left[t^{r} / r !\right] V_{d+1} \xi_{m}^{((d+1) \alpha, r)}=\xi_{d, r, m}^{((d+1) \alpha, r)} .
$$

The $r^{\text {th }}$ incomplete moment of $X$ is defined by

We can write from (7)

$$
\tau_{r}(y)=\int_{-\infty}^{y} x^{r} f(x) d x
$$

$$
\tau_{r}(y)=\left.\gamma\left(1+r \beta^{-1},\left(t^{-1}\right)^{\beta}\right) \sum_{d, m=0}^{\infty} \xi_{d, m}^{((d+1) \alpha, r)}\right|_{[r>-\beta]},
$$


By setting $r=1,2,3$ and 4 we get

$$
\begin{aligned}
& \tau_{1}(y)=\left.\gamma\left(1+\beta^{-1},\left(t^{-1}\right)^{\beta}\right) \sum_{d, m=0}^{\infty} \xi_{d, m}^{((d+1) \alpha, 1)}\right|_{[1>-\beta]}, \\
& \tau_{2}(y)=\left.\gamma\left(1+\beta^{-2},\left(t^{-1}\right)^{\beta}\right) \sum_{d, m=0}^{\infty} \xi_{d, m}^{((d+1) \alpha, 2)}\right|_{[1>-\beta]}, \\
& \tau_{3}(y)=\left.\gamma\left(1+\beta^{-3},\left(t^{-1}\right)^{\beta}\right) \sum_{d, m=0}^{\infty} \xi_{d, m}^{((d+1) \alpha, 3)}\right|_{[3>-\beta]},
\end{aligned}
$$

and

$$
\tau_{4}(y)=\left.\gamma\left(1+\beta^{-4},\left(t^{-1}\right)^{\beta}\right) \sum_{d, m=0}^{\infty} \xi_{d, m}^{((d+1) \alpha, 4)}\right|_{[4>-\beta]} .
$$

\subsection{Moments of residual life}

The $n^{\text {th }}$ moment of the residual life, say

$$
E(X-t)^{n}=\left.z_{n}(t)\right|_{[X>t, n=1,2, \ldots]} \text {, }
$$

uniquely determines $F(x)$. The $n$th moment of the residual life of $X$ is given by

Therefore,

$$
z_{n}(t)=R_{\theta, \alpha, \beta}^{-1}(t) \int_{t}^{\infty}(x-t)^{n} d F_{\theta, \alpha, \beta}(x) .
$$

where

$$
z_{n}(t)=\left.\frac{\gamma\left(1+n \beta^{-1},\left(t^{-1}\right)^{\beta}\right)}{R(t)} \sum_{d, m=0}^{\infty} \kappa_{d, m}^{((d+1) \alpha, n)}\right|_{[n>-\beta]},
$$

and

$$
q_{d+1} \xi_{m}^{((d+1) \alpha, n)}=\kappa_{d, m}^{((d+1) \alpha, n)}
$$

$$
V_{d+1} \sum_{r=0}^{n}(-t)^{n-r}\left(\begin{array}{l}
n \\
r
\end{array}\right)=q_{d+1}
$$

\subsection{Moments of the reversed residual life}

The $n^{\text {th }}$ moment of the reversed residual life, say

we obtain

$$
E(t-X)^{n}=\left.Z_{n}(t)\right|_{[X \leq t, t>0, n=1,2, \ldots]},
$$

$$
Z_{n}(t)=F_{\theta, \alpha, \beta}^{-1}(t) \int_{0}^{t}(t-x)^{n} d F_{\theta, \alpha, \beta}(x) .
$$

Then, the $n^{\text {th }}$ moment of the reversed residual life of $X$ becomes

where

$$
Z_{n}(t)=\left.\frac{\gamma\left(1+n \beta^{-1},\left(t^{-1}\right)^{\beta}\right)}{F(t)} \sum_{d, m=0}^{\infty} \delta_{d, m}^{((d+1) \alpha, n)}\right|_{[n>-\beta]},
$$

and

$$
l_{d+1} \xi_{m}^{((d+1) \alpha, n)}=\delta_{d, m}^{((d+1) \alpha, n)}
$$




$$
V_{d+1} \sum_{r=0}^{n}(-1)^{r}\left(\begin{array}{l}
n \\
r
\end{array}\right) t^{n-r}=l_{d+1}
$$

\subsection{Order statistics}

Suppose $X_{1}:{ }_{n}, X_{2}:{ }_{n}, \ldots, X_{n}: n$, is a random sample (R.S.) from the BHEW model. Let $X_{i}:{ }_{n}$ denote the $i^{\text {th }}$ order statistic. The P.D.F. of $X_{i}: n$ is

$$
f_{i}:{ }_{n}(x)=B^{-1}(i, n-i+1) \sum_{j=0}^{n-i}(-1)^{j}\left(\begin{array}{c}
n-i \\
j
\end{array}\right) f_{\theta, \alpha, \beta}(x) F_{\theta, \alpha, \beta}(x)^{j+i-1} .
$$

Following the result 0.314 of Gradshteyn and Ryzhik (2000) for a power series raised to $n$ is a positive integer we have

$$
\sum_{i=0}^{\infty} c_{n, i} u^{i}=\left.\left(\sum_{i=0}^{\infty} a_{i} u^{i}\right)^{n}\right|_{[n \geq 1]}
$$

where $c_{n, i}$ (for $i=1,2, \ldots$ ) are the coefficients determined from the recurrence Eq. (with $c_{n, 0}=a_{0}^{n}$ )

$$
\left(i a_{0}\right)^{-1} \sum_{m=1}^{i} a_{m} c_{n, i-m}[m(n+1)-i]=c_{n, i}
$$

The P.D.F. of the $i^{t h}$ order statistic of any BHEWmodel can be expressed as

$$
f_{i: n}^{(\theta, \alpha, \beta)}(x)=\sum_{h, d=0}^{\infty} b_{h, d} \pi_{h+d+1}(x)
$$

where

$$
b_{h, d}=n !(h+1)(i-1) !(h+d+1)^{-1} d_{h+1} \sum_{j=0}^{n-i} \frac{(-1)^{j} v_{j+i-1, d}}{(n-i-j) ! j !}
$$

and the quantities $v_{j+i-1, d}$ can be determined with $f_{i+i-1,0}=d_{0}^{j+i-1}$ and recursively for $d \geq 1$

$$
f_{j+i-1, d}=\left(d d_{0}\right)^{-1} \sum_{m=1}^{d} d_{m} v_{j+i-1, d-m}[m(j+i)-d]
$$

4. For the BHEW model

$$
\text { 5. } E X_{i}^{q}: n=\left.\Gamma\left(1+q \beta^{-1}\right) \sum_{h, d, m=0}^{\infty} \quad \xi_{h, d, m}^{(h+d+1, q)}\right|_{[q>-\beta]},
$$

6. where

$$
\text { 7. } b_{h, d} \xi_{m}^{(h+d+1, q)}=\xi_{h, d, m}^{(h+d+1, q)} \text {. }
$$

\section{Estimation}

Let $x_{1}, \ldots, x_{n}$ be a R.S. from the BHEW distribution with parameters $\theta, \alpha$ and $\beta$. Let $\boldsymbol{\Psi}$ be the $3 \times 1$ parameter vector. For getting the maximum likelihood estimates (M.L.E.) of $\boldsymbol{\Psi}$, we have the log-likelihood (L.L.) function 
where

$$
\begin{aligned}
& \ell=\ell(\Psi)=n \log \alpha+n \log \beta+(\beta-1) \sum_{i=1}^{n} \log x_{i}-\sum_{i=1}^{n} x_{i}^{\beta} \\
& -\sum_{i=1}^{n} x_{i}^{\beta}+(\alpha-1) \sum_{i=1}^{n} \log z_{i}+\sum_{i=1}^{n} \log q_{i}+\sum_{i=1}^{n} \log s_{i},
\end{aligned}
$$

and

$$
\begin{gathered}
q_{i}=\left(1-z_{i}^{\alpha}\right)^{\theta-1} /\left[1-\log \left(1-z_{i}^{\alpha}\right)\right]^{2}, \\
s_{i}=\left\{\theta\left[1-\log \left(1-z_{i}^{\alpha}\right)\right]+1\right\}
\end{gathered}
$$

$$
z_{i}=\left[1-\exp \left(-x_{i}^{\beta}\right)\right]
$$

The components of the score vector is available if needed.

\section{Applications}

In this Section, we provide four applications to show empirically its potentiality. We consider the Cramér-Von Mises $\mathrm{W}^{*}$ and the Anderson-Darling $A^{*}$ statistics. The computations are carried out using the $\mathrm{R}$ software. The M.L.E. and the corresponding standard errors (S.E.) (in parentheses) of the new model parameters are given in Tables 2, 4, 6, and 8. The numerical values of the $\mathrm{W}^{*}$ and $\mathrm{A}^{*}$ are listed in Tables 3, 5, 7, and 9. The estimated P.D.F., P-P plot, TTT plot and Kaplan-Meier survival plot of the four data sets of the proposed model are displayed in Figures 3, 4,5 and 6. These four data sets were used for fitting the Odd Lindley EW by Aboraya (2018).

\section{Application 1}

The data consist of 84 observations. The data are: $0.040,1.866,2.385,3.443,0.301$, $1.876,2.481,3.467,0.309,1.899,2.610,3.478,0.557,1.911,2.625,4.570,1.652,2.300$, $3.344,4.602,1.757,3.578,0.943,1.912,2.632,3.595,1.070,1.914,2.646,3.699,1.124$, $1.981,2.661,3.779,1.248,2.010,2.224,3.117,4.485,1.652,2.229,3.166,2.688,3.924$, $1.281,2.038,2.823,4.035,1.281,2.085,2.890,4.121,1.303,2.089,2.902,4.167,1.432$, $4.376,1.615,2.223,3.114,4.449,1.619,2.097,2.934,4.240,1.480,2.135,2.962,4.255$, $1.505,2.154,2.964,4.278,1.506,2.190,3.000,4.305,1.568,2.194,3.103,2.324,3.376$, 4.663. Here, we shall compare the fits of the BHEW distribution with those of other competitive models, namely: Poisson Topp Leone-Weibull (PTL-W), Marshall Olkin extended-Weibull (MOE-W), Gamma-Weibull (Ga-W), Kumaraswamy-Weibull (KwW), Weibull-Fréchet (W-Fr) Beta-Weibull (B-W), Transmuted modified-Weibull, Kumaraswamy transmuted-Weibull (KwT-W), Modified beta-Weibull (MB-W), Mcdonald-Weibull (Mc-W), transmuted exponentiated generalized Weibull distributions, whose P.D.F.s (for $x>0$ ) (for more details about these P.D.F.s see Aboraya (2018)). The parameters of the above densities are all positive real numbers except for the TM-W and TExG-W distributions. Tables 2 list the values of above statistics for seven fitted models. The M.L.E.s and their corresponding standard errors (in parentheses) of the model parameters are also given in these tables. The figures in Table 3 reveal that the 
new distribution yields the lowest values of these statistics and hence provides the best fit to the two data sets.

Table 2: M.L.E.s (S.E. in parentheses) for data set I.

\begin{tabular}{|c|c|}
\hline Model & Estimates \\
\hline $\mathrm{BHEW}_{(\theta, \alpha, \beta)}$ & $\begin{array}{l}814.3149,20.146,0.223 \\
(582.25),(1.683),(0.03)\end{array}$ \\
\hline PTL-W $(\lambda, \alpha, b)$ & $\begin{array}{c}-5.78175,4.22865,0.65801 \\
(1.395),(1.167),(0.039)\end{array}$ \\
\hline MOE-W $(\gamma, \beta, \alpha)$ & $\begin{array}{l}488.8994,0.283246,1261.96 \\
(189.358),(0.013),(351.073)\end{array}$ \\
\hline $\mathrm{Ga}-\mathrm{W}_{(\alpha, \beta, \gamma)}$ & $\begin{array}{l}2.376973,0.848094,3.534401 \\
(0.378),(0.0005296),(0.665)\end{array}$ \\
\hline $\mathbf{K} \mathbf{W}-\mathbf{W}_{(\alpha, \beta, a, b)}$ & $\begin{array}{c}14.4331,0.2041,34.6599,81.8459 \\
(27.095),(0.042),(17.527),(52.014)\end{array}$ \\
\hline $\mathrm{W}-\operatorname{Fr}_{(\alpha, \beta, a, b)}$ & $\begin{array}{c}630.938,0.30,416.097,1.1664 \\
(697.942),(0.032),(232.359),(0.357)\end{array}$ \\
\hline $\mathrm{B}-\mathrm{W}(\alpha, \beta, a, b)$ & $\begin{array}{l}1.36,0.2981,34.1802,11.4956 \\
(1.002),(0.06),(14.838),(6.73)\end{array}$ \\
\hline TM-W $(\alpha, \beta, \gamma, \lambda)$ & $\begin{array}{c}0.2722,1,4.6 \times 10^{-6}, 0.4685 \\
(0.014),\left(5.2 \times 10^{-5}\right),\left(1.9 \times 10^{-4}\right),(0.165)\end{array}$ \\
\hline $\mathrm{KWT}_{\mathrm{W}}-\mathrm{W}_{(\alpha, \beta, \lambda, a, b)}$ & $\begin{array}{c}27.7912,0.178,0.4449,29.5253,168.0603 \\
(33.401),(0.017),(0.609),(9.792),(129.165)\end{array}$ \\
\hline MB-W $(\alpha, \beta, a, b, c)$ & $\begin{array}{l}10.1502,0.1632,57.4167,19.3859,2.0043 \\
(18.697),(0.019),(14.063),(10.019),(0.662)\end{array}$ \\
\hline $\mathrm{Mc}_{\mathrm{c}} \mathrm{W}_{(\alpha, \beta, a, b, c)}$ & $\begin{array}{l}1.9401,0.306,17.686,33.6388,16.7211 \\
(1.011),(0: 045),(6.222),(19.994),(9.722)\end{array}$ \\
\hline TExG-W $(\alpha, \beta, \lambda, a, b)$ & $\begin{array}{c}4.257,0.1532,0.0978,5.23,1173.328 \\
(33.401),(0.017),(0.609),(9.792)\end{array}$ \\
\hline
\end{tabular}


Table 3: $W^{*}$ and $A^{*}$ for data set I.

\begin{tabular}{l|cc}
\hline \hline Model & $W^{*}$ & $A^{*}$ \\
\hline \hline BHEW $_{(\theta, \alpha, \beta)}$ & $\mathbf{0 . 0 9 1 6 5}$ & $\mathbf{0 . 8 7 4 0 9}$ \\
\hline PTL-W $_{(\lambda, \alpha, b)}$ & 0.13967 & 1.19393 \\
\hline MOE-W $_{(\gamma, \beta, \alpha)}$ & 0.39953 & 4.44766 \\
\hline Ga-W $_{(\alpha, \beta, \gamma)}$ & 0.25533 & 1.94887 \\
\hline KW-W $_{(\alpha, \beta, a, b)}$ & 0.18523 & 1.50591 \\
\hline W-Fr $_{(\alpha, \beta, a, b)}$ & 0.25372 & 1.95739 \\
\hline B-W $_{(\alpha, \beta, a, b)}$ & 0.46518 & 3.21973 \\
\hline TM-W $_{(\alpha, \beta, \gamma, \lambda)}$ & 0.80649 & 11.20466 \\
\hline MEx-W $_{(\alpha, \beta, a, b, c)}$ & 0.47172 & 3.26561 \\
& & \\
\hline \hline$(\alpha, \beta, \lambda, a, b)$ & 0.16401 & 1.36324 \\
\hline
\end{tabular}



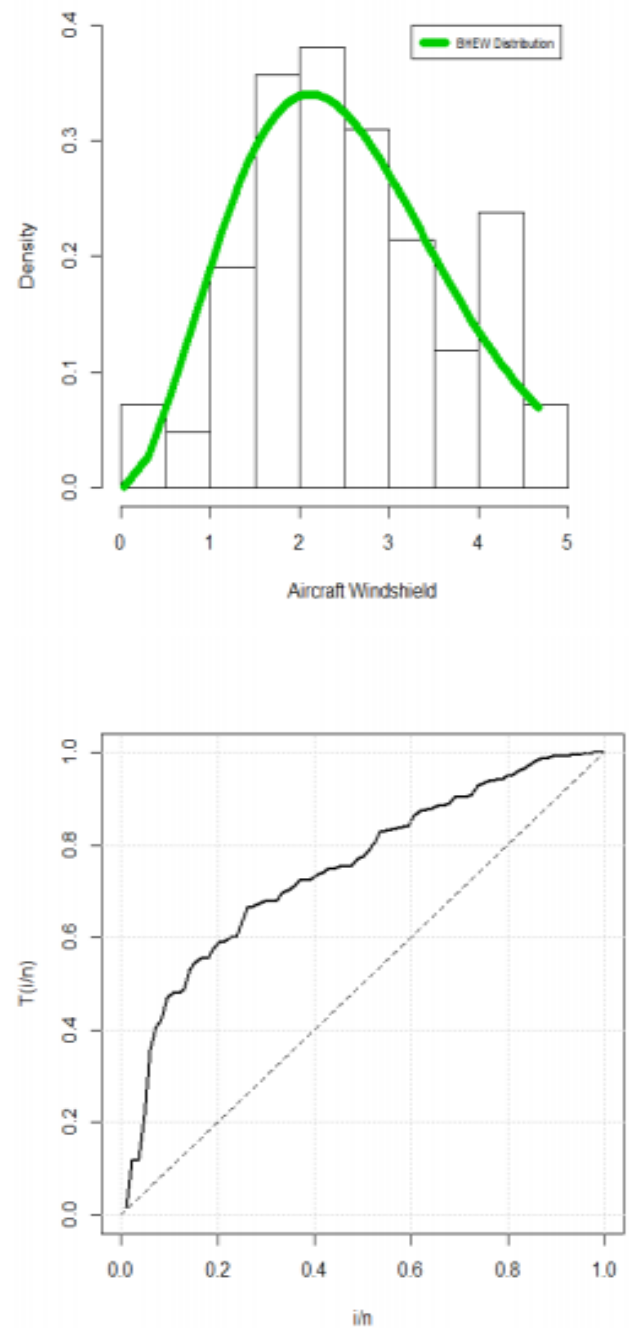
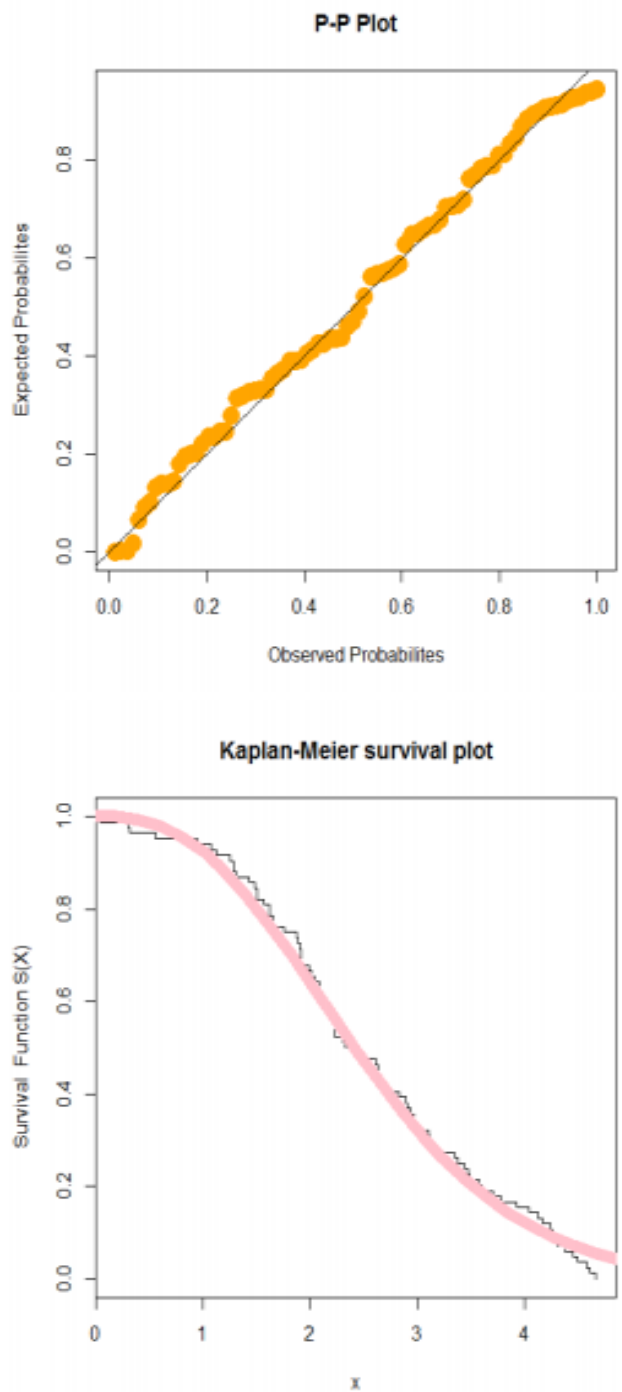

Figure 3: Estimated P.D.F., P-P plot, TTT plot and Kaplan-Meier survival plot for data set I.

\section{Application 2}

This data set represents the remission times (in months) of a random sample of 128 bladder cancer patients. This data is given by: 0.080, 2.090, 3.48, 4.87, 6.940, 8.66, $13.110,23.63,0.200,2.23,3.52,4.980,6.97,9.020,13.29,0.400,2.26,3.57,5.060,7.09$, $9.220,13.800,25.74,0.500,2.46,3.640,5.09,7.260,9.47,14.24,25.820,0.510,2.54$, $3.70,5.170,7.28,9.740,14.760,26.310,0.81,2.620,3.820,5.320,7.320,10.060,14.770$, $32.150,2.64,3.88,5.320,7.39,10.34,14.830,34.26,0.90,2.690,4.18,5.340,7.59$, $10.660,15.96,36.660,1.05,2.690,4.23,5.410,7.62,10.750,16.62,43.010,1.190,2.750$, $4.260,5.410,7.63,17.120,46.12,1.260,2.83,4.330,5.49,7.660,11.25,17.140,79.05$, $1.350,2.870,5.620,7.870,11.640,17.360,1.40,3.02$, 4.340, 5.710, 7.93, 11.790, 18.10, $1.460,4.400,5.85,8.260,11.98,19.130,1.76,3.250,4.50,6.250,8.37,12.020,2.020$, $3.31,4.51,6.54,8.53,12.030,20.28,2.020,3.36,6.760,12.07,21.730,2.07,3.36,6.930$, $8.65,12.63,22.690$. We compare the fits of the BHEW distribution with other competitive models, namely: the TMW, MBW, transmuted additive Weibull distribution (TA-W), exponentiated transmuted generalized Rayleigh (ETGR) and the W distributions 
with corresponding densities (for $\mathrm{x}>0$ ) (for more details about these P.D.F.s see Aboraya (2018)).

Table 4: M.L.E.s (S.E. in parentheses) for data set II.

\begin{tabular}{lc}
\hline \hline Model & Estimates \\
\hline BHEW $_{(\theta, \alpha, \beta)}$ & $61.746,45.807,0.170$ \\
& $(157.0984),(7.291,(0.0544)$ \\
W $_{(\alpha, \beta)}$ & $(0.853),(0.068)$ \\
& $0.1208,0.8955,0.0002,0.2513$ \\
TM-W $_{(\alpha, \beta, \gamma, \lambda)}$ & $(0.024),(0.626),(0.011),(0.407)$ \\
& $0.1502,0.1632,57.4167,19.3859,2.0043$ \\
MB-W $_{(\alpha, \beta, a, b, c)}$ & $(22.437),(0.044),(37.317),(13.49),(0.789)$ \\
& $(0.032),(0.125),\left(6.106 \times 10^{-3}\right),(0.035),(0.28)$ \\
TA-W $_{(\alpha, \beta, \gamma, \theta, \lambda)}$ & $0.1139,0.9722,3.0936 \times 10^{-5}, 1.0065,-0.163$ \\
& $(5.389),\left(3.965 \times 10^{-3}\right),(0.036),(0.26)$ \\
\hline ETG- $_{(\alpha, \beta, \delta, \lambda)}$ & \\
\hline \hline
\end{tabular}

Table 5: $W^{*}$ and $A^{*}$ for data set II.

\begin{tabular}{|c|c|c|}
\hline Model & $W^{*}$ & $A^{*}$ \\
\hline $\operatorname{BHEW}_{(\theta, \alpha, \beta)}$ & 0.09548 & 0.5947 \\
\hline $\mathrm{W}_{(\alpha, \beta)}$ & 0.10553 & 0.66279 \\
\hline TM-W $(\alpha, \beta, \gamma, \lambda)$ & 0.12511 & 0.76028 \\
\hline MB-W $W_{(\alpha, \beta, a, b, c)}$ & 0.10679 & 0.72074 \\
\hline TA-W ${ }_{(\alpha, \beta, \gamma, \theta, \lambda)}$ & 0.11288 & 0.70326 \\
\hline ETG-R $\mathbf{R}_{(\alpha, \beta, \delta, \lambda)}$ & 0.39794 & 2.36077 \\
\hline
\end{tabular}



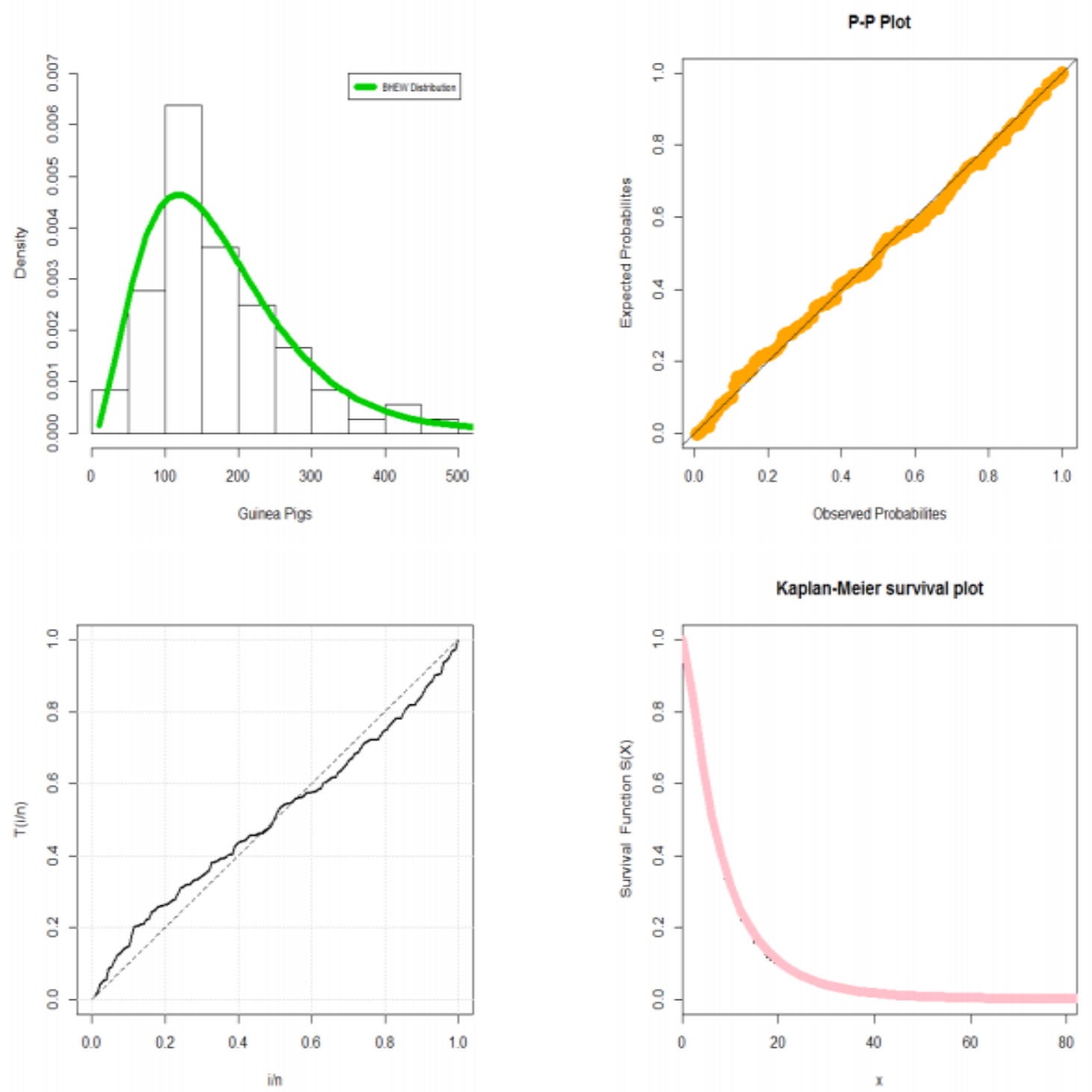

Figure 4: Estimated P.D.F., P-P plot, TTT plot and Kaplan-Meier survival plot for data set II.

\section{Application 3}

The second real data set corresponds to the survival times (in days) of 72 guinea pigs infected with virulent tubercle bacilli reported by Bjerkedal (1960). The data are: 72,74, $77,10,33,44,56,59,92,93,96,107,107,108,108,100,100,102,105,108,116,120$, $121,122,109,112,113,115,122,124,130,146,153,159,160,134,136,139,144,163$, $163,168,176,183,195,196,215,216,222,230,197,202,213,231,240,245,293,327$, $342,251,253,254,255,278,347,361,402,171,172,432,458,555$.

We shall compare the fits of the BHEW distribution with those of other competitive models, namely: the Weibull-Weibull (W-W), the Odd Weibull-Weibull (OW-W), the gamma exponentiated-exponential (GaE-E) distributions, whose P.D.F.s (for $\mathrm{x}>0$ ) (for more details about these P.D.F.s see Aboraya (2018)). 
Table 6: M.L.E.s (S.E. in parentheses) for data set III.

\begin{tabular}{lc}
\hline \hline Model & Estimates \\
\hline \hline BHEW $_{(\theta, \alpha, \beta)}$ & $10.257,11.369,0.2267$ \\
& $(16.611),(2.99),(0.08)$ \\
& $2.6594,0.6933,0.0270$ \\
W-W & $(0.7129),(0.1707),(0.0193)$ \\
& $11.1576,0.0881,0.4574$ \\
OW-W & $(4.5449)(0.0355)(0.0770)$ \\
& $2.1138,2.6006,0.0083$ \\
$\operatorname{GaE}_{(\beta, \gamma, \lambda)}$ & $(1.3288),(0.5597),(0.0048)$ \\
\hline \hline
\end{tabular}

Table 7: $W^{*}$ and $A^{*}$ for data set III.

\begin{tabular}{l|cc}
\hline \hline Model & $W^{*}$ & $A^{*}$ \\
\hline \hline BHEW $_{(\theta, \alpha, \beta)}$ & $\mathbf{0 . 0 4 1 8}$ & $\mathbf{0 . 2 7 9 2}$ \\
& & \\
\hline $\mathrm{W}-\mathrm{W}_{(\beta, \gamma, \lambda)}$ & 0.1427 & 0.7811 \\
\hline OW-W & & \\
& & \\
\hline $\operatorname{GaE}_{(\beta, \gamma, \lambda)}$ & 0.4494 & 2.4764 \\
& & \\
& 0.3150 & 1.7208 \\
\hline \hline
\end{tabular}



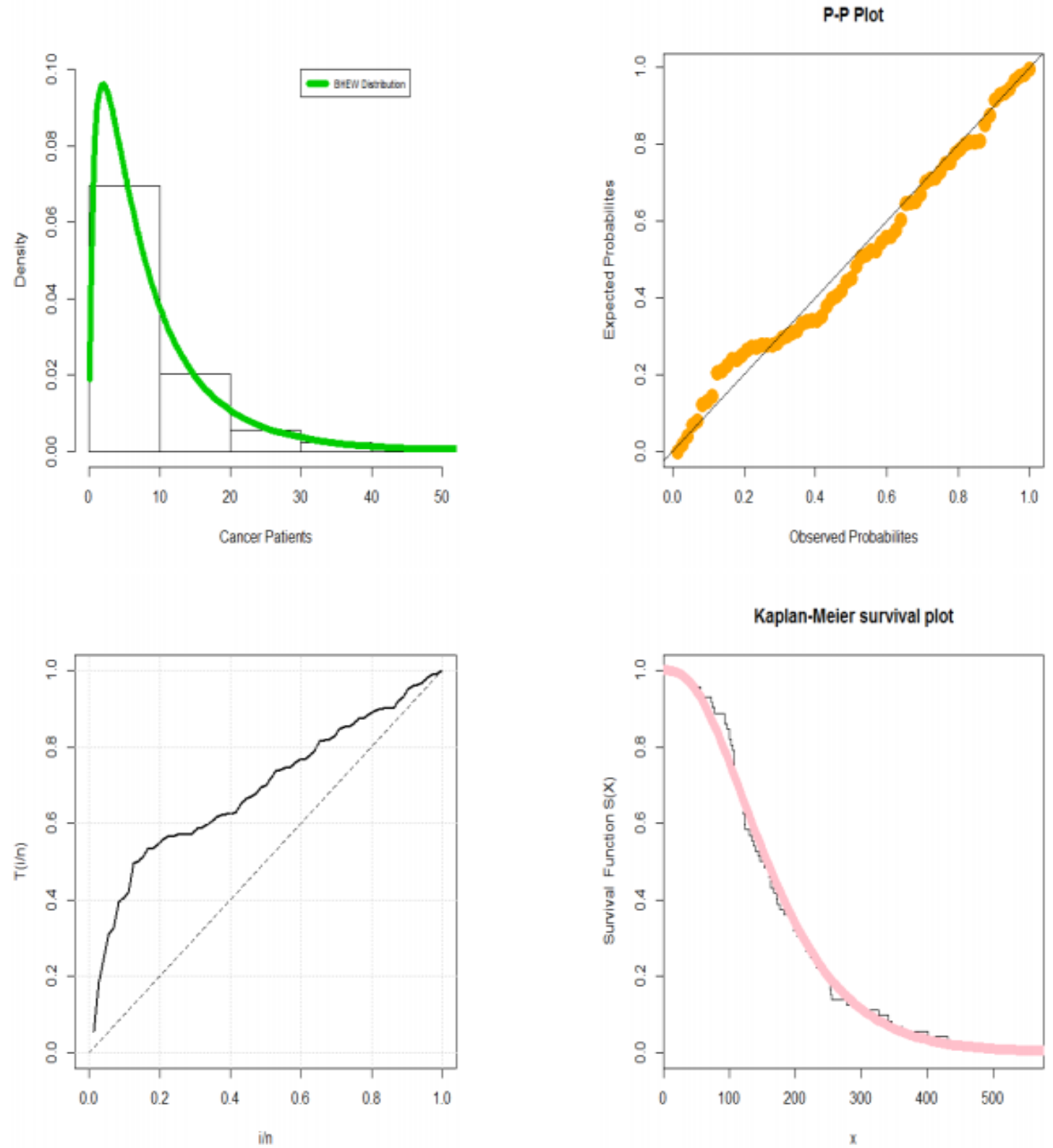

Figure 5: Estimated P.D.F., P-P plot, TTT plot and Kaplan-Meier survival plot for data set III.

\section{Application 4: Glass fibers data}

This data consists of 63 observations of the strengths of $1.5 \mathrm{~cm}$ glass fibers, originally obtained by workers at the UK National Physical Laboratory. The data are: 0.550, 0.74, $0.770,0.81,0.840,0.93,1.040,1.11,1.130,1.240,1.250,1.27,1.280,1.29,1.300,1.36$, $1.390,1.42,1.480,1.48,1.490,1.49,1.500,1.50,1.510,1.52,1.530,1.54,1.550,1.55$, $1.580,1.590,1.60,1.610,1.610,1.6100,1.61,1.620,1.62,1.630,1.64,1.660,1.66$, $1.660,1.67,1.68,1.680,1.69,1.700,1.70,1.730,1.76,1.760,1.77,1.780,1.81,1.820$, $1.84,1.840,1.890,2.00,2.01,2.240$. For this data set, we shall compare the fits of the BHEW distribution with some competitive models like EW, T-W and OLL-W (for more details about these P.D.F.s see Aboraya (2018)). 
Table 8: M.L.E.s (S.E. in parentheses) for data set IV.

\begin{tabular}{lc}
\hline \hline Model & Estimates \\
\hline BHEW $_{(\theta, \alpha, \beta)}$ & $908.64,21.127,0.5$ \\
& $(577.313),(1.610),(0.057)$ \\
EW $_{(a, \alpha, \beta)}$ & $0.671,7.285,1.718$ \\
& $(0.249),(1.707),(0.086)$ \\
T-W $_{(a, \alpha, \beta)}$ & $-0.5010,5.1498,0.6458$ \\
& $(0.2741),(0.6657),(0.0235)$ \\
OLL-W $_{(\theta, \alpha, \beta)}$ & $0.9439,6.0256,0.6159$ \\
& $(0.2689),(1.3478),(0.0164)$ \\
\hline \hline
\end{tabular}

Table 9: $W^{*}$ and $A^{*}$ for data set IV.

\begin{tabular}{l|cc}
\hline \hline Model & $W^{*}$ & $A^{*}$ \\
\hline \hline BHEW $_{(\theta, \alpha, \beta)}$ & $\mathbf{0 . 3 1 6 1}$ & $\mathbf{1 . 7 3 0 1}$ \\
\hline $\mathrm{EW}_{(a, \alpha, \beta)}$ & 0.636 & 3.484 \\
\hline $\mathrm{T}-\mathrm{W}_{(a, \alpha, \beta)}$ & 1.0358 & 0.1691 \\
\hline OLL-W $_{(\theta, \alpha, \beta)}$ & 1.2364 & 0.2194 \\
& & \\
\hline \hline
\end{tabular}



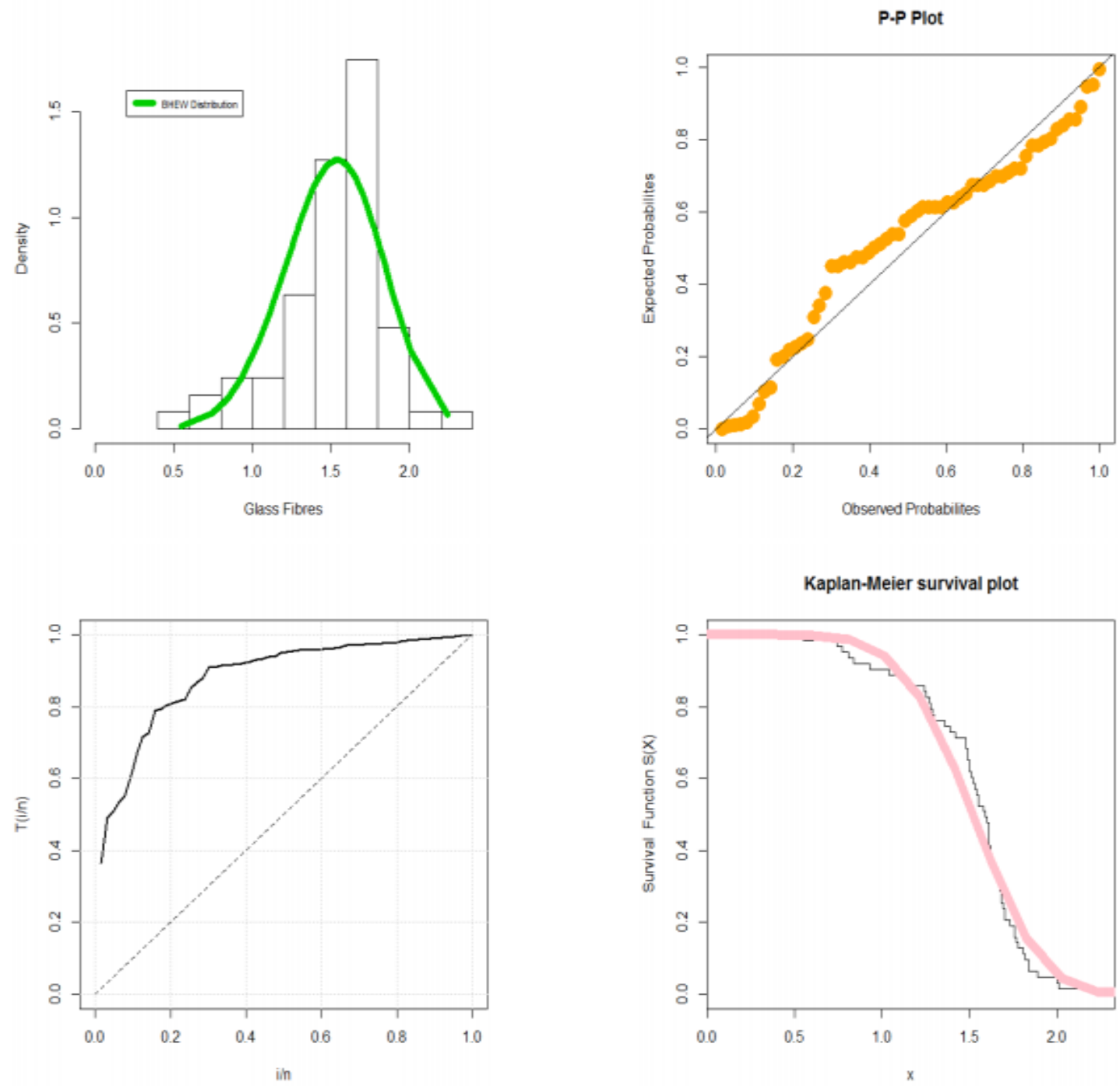

Figure 6: Estimated P.D.F., P-P plot, TTT plot and Kaplan-Meier survival plot for data set IV.

Based on Tables 3, 5, 7 and 9, the BHEW lifetime model provides adequate fits as compared to other Weibull models with small values for $W^{*}$ and $A^{*}$. The BHEW lifetime model is better than the PTL-W, MOE-W, W-Fr, B-W, TM-W, KwT-W, Ga-W, $\mathrm{Kw}-\mathrm{W}, \mathrm{MB}-\mathrm{W}, \mathrm{Mc}-\mathrm{W}$ and TExG-W models in modeling the failure times data, also the new model is much better than the TL-E, W, TM-W, MB-W, TA-W and ETG-R models in modeling cancer patients data, and much better than the OW-W and GaE-E models in modeling survival times of Guinea pigs. Finally, the proposed model is much better than the EW, T-W and OLL-W models in modeling glass fibers data. Also, Plots of estimated P.D.F., P-P, TTT and Kaplan-Meier survival given in Figures 3-6 supports these results.

\section{Concluding remarks}

A new three-parameter lifetime model called the Burr-Hatke exponentiated Weibull (BHEW) model is introduced. The main justification for the practicality of the BHEW lifetime model is based on the wider use of the Weibull and exponentiated Weibull 
models. We are also motivated to introduce the BHEW lifetime model since it exhibits increasing, decreasing and bathtub hazard rates. The BHEW model can be viewed as a mixture of the EW density. It can also be considered as a suitable model for fitting the left skewed, right skewed, symmetric, and unimodal data. We prove empirically the great importance and wide flexibility of the BHEW model in modeling four types of lifetime data, the new model provides adequate fits as compared to other Weibull models with small values for $\mathrm{W}^{*}$ and $\mathrm{A}^{*}$ so the new model is much better than other competitive model in modeling four data sets. The proposed lifetime model is much better than the Poisson Topp Leone-Weibull, Marshall Olkin extended-Weibull, Gamma-Weibull, Kumaraswamy-Weibull, Weibull-Fréchet, B-W, Beta-Weibull, Kumaraswamy transmuted-Weibull, transmuted modified-Weibull, transmuted exponentiated generalized Weibull, modified beta-Weibull and McDonald-Weibull models in modeling the failure times data. In modeling cancer patient's data, the new model is much better than the transmuted linear exponential, Weibull, Transmuted modified-Weibull, modified beta-Weibull, transmuted additive-Weibull and exponentiated transmuted generalized Rayleigh models. The BHEW model is much better than the Weibull-Weibull, Odd Weibull-Weibull and gamma exponentiated-exponential models in modeling survival times of Guinea pigs. Finally, the BHEW model is better than transmuted-Weibull, the exponentiated-Weibull and Odd Log Logistic-Weibull models in modeling glass fibers data.

\section{References}

1. Aboraya, M. (2018). A new flexible lifetime model with statistical properties and applications, Pak.j.stat.oper.res, forthcoming.

2. Almamy, J. A., Ibrahim, M., Eliwa, M. S., Al-mualim, S. and Yousof, H. M. (2018). The two-parameter odd Lindley Weibull lifetime model with properties and applications International Journal of Statistics and Probability, 7(4), 19277040.

3. Aryal, G. R., Ortega, E. M., Hamedani, G. G. and Yousof, H. M. (2017). The Topp Leone Generated Weibull distribution: regression model, characterizations and applications, International Journal of Statistics and Probability, 6, 126-141.

4. Alzaatreh, A., Lee, C. \& Famoye, F. (2013). A new method for generating families of continuous distributions, Metron, 71, 63-79.

5. Bjerkedal, T. (1960). Acquisition of resistance in guinea pigs infected with different doses of virulent tubercle bacilli. American Journal of Hygiene, 72, 130148.

6. Bourguignon, M., Silva, R.B. \& Cordeiro, G.M. (2014). The Weibull--G family of probability distributions, Journal of Data Science, 12, 53-68.

7. Brito, E., Cordeiro, G. M., Yousof, H. M., Alizadeh, M. and Silva, G. O. (2017). Topp-Leone Odd Log-Logistic Family of Distributions, Journal of Statistical Computation and Simulation, 87(15), 3040-3058.

8. Cordeiro, G. M., Yousof, H. M., Ramires, T. G. and Ortega, E. M. M. (2018). The Burr XII system of densities: properties, regression model and applications. 
Journal of Statistical Computation and Simulation, 88(3), 432-456.

9. Gradshteyn, I. S. and Ryzhik, I. M. (2000). Table of Integrals, Series and Products (sixth edition). San Diego: Academic Press.

10. Hamedani G. G. Yousof, H. M., Rasekhi, M., Alizadeh, M., Najibi, S. M. (2017). Type I general exponential class of distributions. Pak. J. Stat. Oper. Res., XIV (1), 39-55.

11. Korkmaz, M. C., Altun, E., Yousof, H. M. and Hamedani G. G. (2019). The Odd Power Lindley Generator of Probability Distributions: Properties, Characterizations and Regression Modeling, International Journal of Statistics and Probability, 8(2). 70-89.

12. Lee, C., Famoye, F. \& Olumolade, O. (2007). Beta-Weibull distribution: some properties and applications to censored data. Journal of Modern Applied Statistical Methods, 6, 17.

13. Maniu, A. I. and Voda, V. G. (2008). Generalized Burr-Hatke Equation as Generator of a Homogaphic Failure rate, Journal of applied quantitative methods, 3, 215-222.

14. Mudholkar, G. S. \& Srivastava, D. K. (1993). Exponentiated Weibull family for analyzing bathtub failure-rate data. IEEE Transactions on Reliability, 42, 299302.

15. Mudholkar, G. S., Srivastava, D. K. \& Freimer, M. (1995). The exponentiated Weibull family: A reanalysis of the bus-motor-failure data. Technometrics, 37, 436-445.

16. Nadarajah, S., Cordeiro, G. M. \& Ortega, E. M. M. (2013). The exponentiated Weibull distribution: A survey, Statistical Papers, 54, 839-877.

17. Weibull, W. (1951). Statistical distributions function of wide applicability. J. Appl. Mech.-Trans, 18(3), 293-297.

18. Yousof, H. M., Afify, A. Z., Alizadeh, M., Butt, N. S., Hamedani, G. G. and Ali, M. M. (2015). The transmuted exponentiated generalized-G family of distributions, Pak. J. Stat. Oper. Res., 11 (4), 441-464.

19. Yousof, H. M., Afify, A. Z., Alizadeh, M., Butt, N. S., Hamedani, G. G. and Ali, M. M. (2015). The transmuted exponentiated generalized-G family of distributions, Pak. J. Stat. Oper. Res., 11 (4), 441-464.

20. Yousof, H. M., Afify, A. Z., Cordeiro, G. M., Alzaatreh, A., and Ahsanullah, M. (2017). A new four-parameter Weibull model for lifetime data. Journal of Statistical Theory and Applications, 16(4), 448 - 466.

21. Yousof, H. M., Altun, E., Ramires, T. G., Alizadeh, M. and Rasekhi, M. (2018). A new family of distributions with properties, regression models and applications, Journal of Statistics and Management Systems, 21(1), 163-188. 


\section{Appendix}
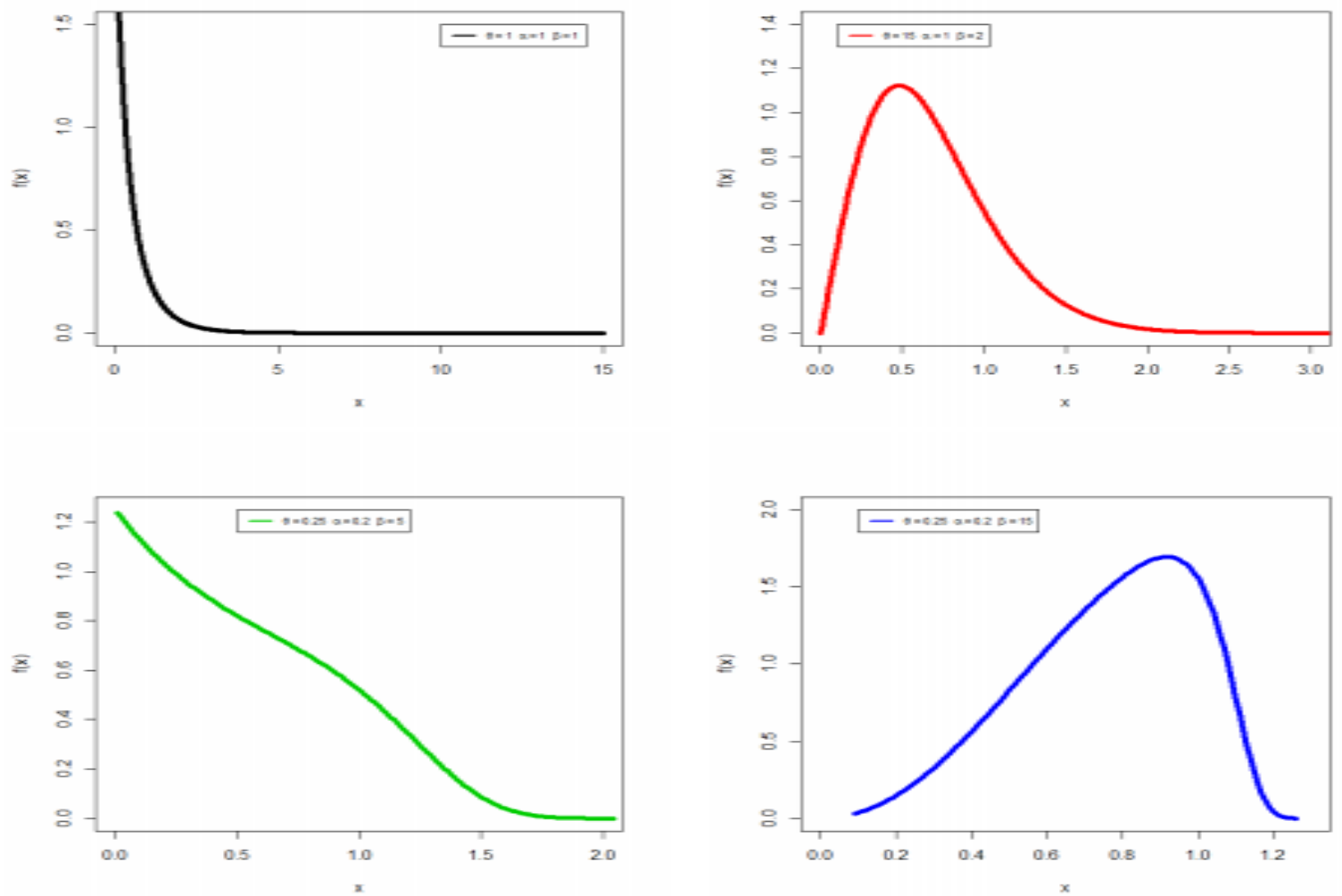

Figure 1: Plots of the BHEW P.D.F.
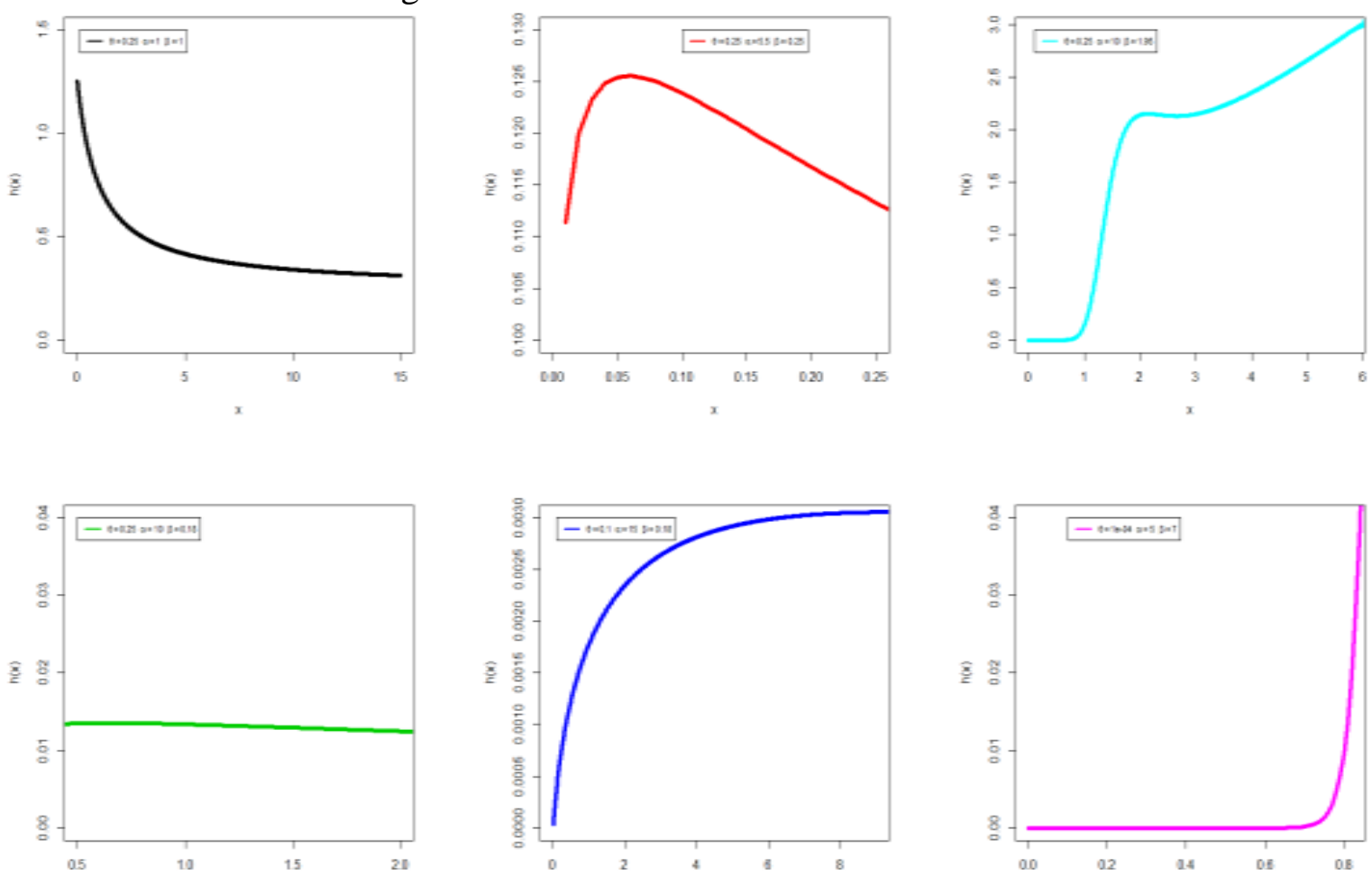

Figure 2: Plots of the BHEW H.R.F. 